\title{
H Reflex from Soleus Muscle After Ankle Sprain of a College Basketball Player
}

\author{
Daikuya $\mathrm{S}^{1 *}$, Ono $\mathrm{A}^{1}$ and Yabe $\mathrm{K}^{2}$ \\ ${ }^{1}$ Department of Rehabilitation Kishiwada Eishinkai Hospital, Japan \\ ${ }^{2}$ Nagoya University, Japan
}

\begin{abstract}
To clarify that the fluctuation of the excitability of spinal neural function after ankle sprain, we studied the alteration of $\mathrm{H}$ reflex from the soleus muscle in a patient with ankle sprain.

A Subject was male college basketball player, whose age was twenty-one years old, and diagnosed left ankle inversed sprain (grade II). $\mathrm{H}$ reflex and maximal $\mathrm{M}$ wave from the bilateral soleus muscle were evoked at rest and amplitude ratio of $\mathrm{H} / \mathrm{Mmax}$ was calculated. And, amplitude ratio of $\mathrm{H} / \mathrm{Mmax}$ was compared between after and before injury.

As a result of this study, amplitude ratio of $\mathrm{H} / \mathrm{Mmax}$ was increased at three days after ankle sprain on both injured side and non-injured side compared with both before and one month after injury and, Long Latency Reflex (LLR) was appeared behind the $\mathrm{H}$ reflex consecutively on the only injured side.

From results of this study, it was cleared that the excitability of spinal and supra-spinal neural function related to soleus muscle at three days after was increased due to pain, joint effusion and/or joint instability.
\end{abstract}

Keywords: H reflex; Ankle sprain; Long latency reflex

\section{Introduction}

Electrophysiological evaluation has been often used in the field of neurological science and clinical neurology. In the field of rehabilitation medicine, electrophysiological findings were often used for an expectation of a prognosis of stroke patients. On the other hand, in the field of sport science and orthopedic rehabilitation medicine, the application of electrophysiological findings was increased for functional evaluation in the patient with sport injury [1-3]. We think electrophysiological findings are useful in the patient with orthopedic patient, because even as orthopedic patients, they surely have neuromuscular impairment under adaptation process for their activity of daily life, occupation, recreation or sport activity against their soreness, instability and so on. And more, as a functional evaluation after sport injury, Range of Motion (ROM) and muscular strength are main indices. However, the recovery of body movement and wound healing level always does not match with them. Therefore, we think though patients' ROM and muscular strength was not enough, they may reach to competition return without unstable and strange feeling in the sport activity. Another hand, some patients after sport injury acquired only insufficient sport activity under the enough recovery of ROM and muscular strength. From our clinical impression, as the functional recovery index, only ROM and muscular strength is not enough. So, we have a consideration that electrophysiological measure may be useful in the functional evaluation which gives the suggestion which is sufficient for the competition return after sport injury. Therefore, we always use the electrophysiological test as the physical therapeutic evaluation in the patient with sport injury.

In this study, we acquired a peculiar finding of soleus $\mathrm{H}$ reflex in the patient with ankle sprain during a basketball.

\section{Materials and Methods}

Subject was 21-year-old male college basketball player. His diagnosis was left ankle inversed sprain (grade II). There were swelling, pain and instability on injured side ankle. And, an over contraction of the surrounding muscle of ankle was existed bilaterally. Muscle strength could not be measured due to ankle motion pain. As for the activity of daily life, walking in pain was appeared, and going up and down stairs was needed too much time. Running and jumping were impossible. Prior to the experiment, a subject was informed the purpose of this study based on the descriptions, and his agreement for participation was obtained from the subject.

$\mathrm{H}$ reflex from soleus muscle was recorded at three times, which were before injury (medical check), three days after injury and one month after injury. $\mathrm{H}$ reflex from soleus muscle were evoked by single stimulation to tibial nerve at the popliteal fossa on prone position at rest. The stimulating conditions for recording the $\mathrm{H}$ reflex was as follows; intensity of an appearance threshold of $\mathrm{M}$ wave, which was identified with a gain of $500 \mu \mathrm{V} / \mathrm{div}$ on a screen, duration of $1.0 \mathrm{~ms}$, frequency of $0.5 \mathrm{~Hz}$ and numbers of 16 times. As to the recording conditions of $\mathrm{H}$ reflex, recording electrode was placed on soleus muscle (leg medial and 4 or $5 \mathrm{~cm}$ upper from ankle joint) and reference electrode was put on the ipsilateral attachment of Achilles tendon (calcaneus). Sweep time on recording was $200 \mathrm{~ms}$. The raw data were amplified with a band pass between $20 \mathrm{~Hz}$ and $2000 \mathrm{~Hz}$ by a Nicolet Viking Quest. As the amplitude of H-reflex, the mean value of the peak to peak amplitude of 16 times was calculated, and the amplitude ratio of $\mathrm{H} / \mathrm{Mmax}$ was found out due to mean amplitude of $\mathrm{H}$ reflex was divided by amplitude of maximal M-wave. Amplitude ratio of H/Mmax was compared with among before injury, three days after injury and one month after injury.

*Corresponding author: Shinichi Daikuya, Department of Rehabilitation, Kishiwada Eishinkai Hospital, 1-12-1 Nakai-cho, Kishiwada-City, Osaka, 596-0003 Japan, Tel:+81-72-443-0081; Fax: +81-72-443-4614; E-mail: s_daikuya@eishinkaihsp.or.jp.

Received April 17, 2013; Accepted July 25, 2013; Published July 27, 2013

Citation: Daikuya S, Ono A, Yabe K (2013) H Reflex from Soleus Muscle After Ankle Sprain of a College Basketball Player. J Trauma Treat 2: 175. doi:10.4172/2167. 1222.1000175

Copyright: (c) 2013 Daikuya S, et al. This is an open-access article distributed under the terms of the Creative Commons Attribution License, which permits unrestricted use, distribution, and reproduction in any medium, provided the original author and source are credited. 


\section{Results}

Typical waveforms acquired in this study were displayed on (Figure 1). Amplitude ratio of $\mathrm{H} / \mathrm{Mmax}$ on before injury, three days after injury and one month after injury were $0.17,0.88,0.21$ on noninjured side, and $0.62,1.23,0.58$ on injured side, respectively (Table 1). Bilaterally, amplitude ratio of $\mathrm{H} / \mathrm{Mmax}$ was increased at three days after injury. In addition, a rhythmical waveform was observed followed by an appearance of $\mathrm{H}$ reflex after three days injury on injured side (Figure 2).

\section{Discussion}

$\mathrm{H}$ reflex was a useful measure to examine the excitability of spinal neural function and muscle activation [4-6]. It was applied not only in the patient with neural disorder but also in the field of musculoskeletal injury and sport science [7-9]. Amplitude of H-reflex was the index of a validation of excitability of spinal neural function and muscle activation. However, $\mathrm{H}$ reflex was easy to fluctuate with subjects' muscle condition. So, amplitude ratio H/MMAX was studied in this study as a parameter of spinal neural function [10].

As for the rhythmical waveform appeared after the $\mathrm{H}$ reflex on three days after injury on injured side was thought to be Long Latency Reflex (LLR). We have reported in previous study similar phenomenon [2]. LLR in the lower extremities is affected by the excitability of the spinal cord, brainstem or motor cortex $[11,12]$. Accordingly, in this case, a transient increase of excitability of spinal neural function on both sides after three days from injury was appeared. And more, on only injured side, it was cleared that the excitability of supra-spinal neural function was increased.

In this case, it was cleared that the excitability of spinal neural function related to soleus muscle was increased at three days after injury on both sides. In addition, LLR was appeared three days after injury on injured side. As for these findings related to alteration of spinal and supra spinal neural function in this study, it must be considered from following two viewpoints of the direct effect caused by injury and the indirect effect acquired in the process of adapting activity of daily life with taking care of his foot after injury. Firstly, the participation of the swelling and the pain were thought as the direct effect due to ankle sprain. Palmieri et al (2004) [13] has reported that the $\mathrm{H}$ reflex of the peroneus longus muscle, the tibialis anterior, and the soleus

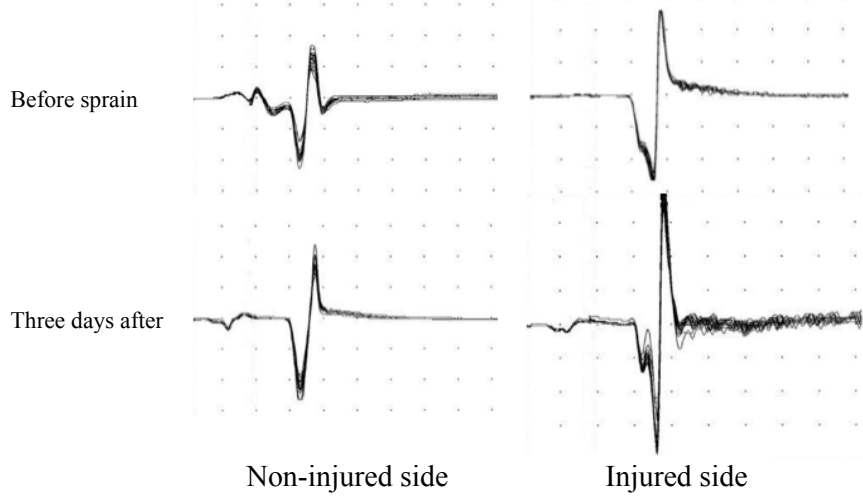

Figure 1: $\mathrm{H}$ reflex from soleus muscle before and after ankle sprain Superimposed raw waves of 16 times recording are displayed. $\mathrm{H}$ reflex had been recorded reproducibility.

$\mathrm{H}$ reflex was enlarged at three days after ankle sprain bilaterally. Amplitude sensitivity: $1 \mathrm{mV} / \mathrm{div}$, sweep: $10 \mathrm{~ms} / \mathrm{div}$.

\begin{tabular}{|c|c|c|c|}
\hline & Before & Three Days after & One Month later \\
\hline Non-injured side & 0.17 & 0.88 & 0.21 \\
\hline Injured side & 0.62 & 1.23 & 0.58 \\
\hline
\end{tabular}

Amplitude ratio of $\mathrm{H} / \mathrm{M}$ was increased at the three days after ankle sprain on both injured side and non injured compared with both before and one month after injury. An effect from ankle sprain have not remained since one month after ankle sprain

Table 1: The alteration of amplitude ratio of $\mathrm{H} / \mathrm{M}$.

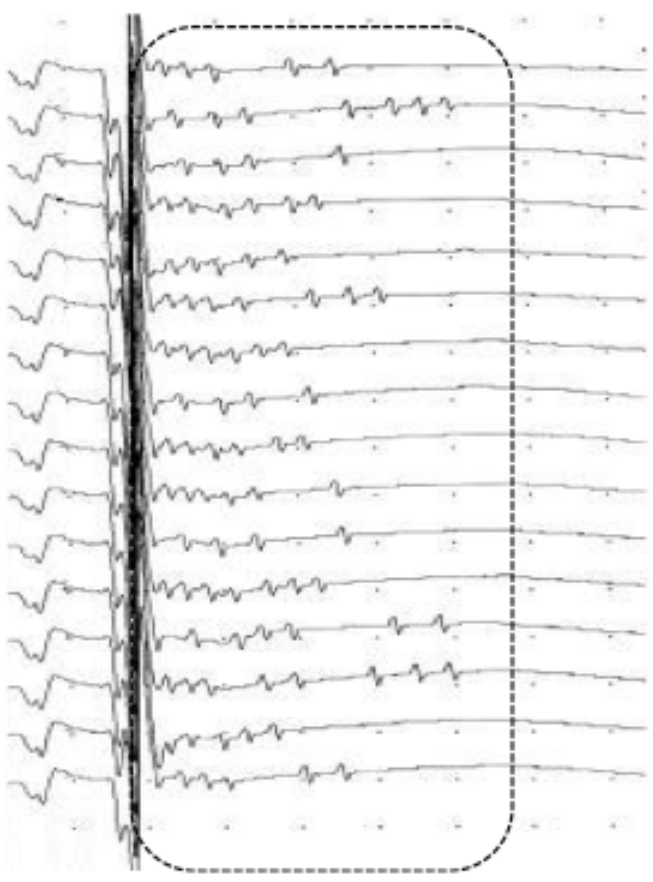

Figure 2: $\mathrm{H}$ reflex from soleus muscle at three days after ankle sprain tfollowed by an appearance of $\mathrm{H}$ reflex on injured side (enclosure part by broken line). Amplitude sensitivity: $1 \mathrm{mV} / \mathrm{div}$ and sweep: $20 \mathrm{~ms} / \mathrm{div}$.

muscle were enlarged by the swelling around the ankle joint and the reduction of the recurrent inhibition caused by an ankle joint effusion and an ankle pain. In this study, spinal reflex was also facilitated by a slight swelling of ankle and joint pain. Next, an involvement of ankle instability was enumerated as in direct effect after ankle sprain [14] has reported that $\mathrm{H}$ reflex of soleus muscle and peroneus longus muscle decreased by inhibitive effect of the joint origin (arthrogenic muscle inhibition) in patients with ankle instability. In this case, it was thought that the facilitative effect to spinal neural function due to an increase of tonic muscle contraction of around the ankle as the defensive reaction for avoiding the pain and/or instability was larger than the inhibitive effect above mentioned, because he has already adapted to activity of his daily life in the situation of his swelling, pain and instability with over contraction of the surrounding muscle of ankle. After all, though those findings, which seemed that the excitability of a supra-spinal neural function increases on three days after injury, was obtained, for this case, it was thought that the facilitation of the spinal reflex had occurred because tonic contraction of the surroundings muscle of ankle joint such as the soleus muscles repeated the posture maintenance and the management for an instability and the pain of ankle joint at an immediate phase of ankle sprain. Moreover, an arthrogenic inhibitory effect and spinal inhibition due to joint instability have not remained in this case because an amplitude ratio of $\mathrm{H} / \mathrm{Mmax}$ acquired at one month after injury has become the same state of before injury. 
Generally, an amplitude ration of H/Mmax does not exceed 1.0, because the Mmax, which is amplitude of maximal $M$ wave, means compound action potential, because of which is summation of all the motor units excited by nerve stimulation. However, the value of 1.23 was obtained in this study. This is considered that because it could not stimulate by greater than $100 \mathrm{~mA}$ with a set of electromyography equipment, supra-maximal $M$ wave might not be elicited. However, because it should be noted that it is larger than that of other recording, it may have been some kind of change for nerve conduction and excitability of muscle membrane cannot be denied. But, in order to increase the accuracy of the data, it is necessary to reconsider the configuration of the electromyography equipment. And, in this study, pre-stimulus background electromyography was not recorded, and ankle fixation was not regulated on recording the $\mathrm{H}$ reflex. These points are the limitation of this study, and it needed to be considered in the future.

\section{Conclusion}

The excitability of spinal neural function related to soleus muscle at three days after injury was increased, and this factor was the effect of pain and/or joint effusion, and adaptation to activities of daily life with painful and unstable ankle due to ankle sprain. And the influence of increase of excitability of spinal neural function on injured side spread to non-injured side. Moreover, on the injured side, supra-spinal function was affected with both the alteration of excitability of spinal neural function and the adaptation to painful and unstable activities of daily life. At one month after injury, their influences were vanished and the inhibitive effect to spinal neural function due to ankle instability was disappeared.

Even if a slight injury of remote part like ankle sprain, the neural function has to be paid attention at least one month after injury in the field of orthopedic physical therapy because the alteration of the excitability of spinal neural function was existed for one month after injury.

\section{References}

1. Héroux ME, Tremblay $F$ (2006) Corticomotor excitability associated with unilateral knee dysfunction secondary to anterior cruciate ligament injury. Knee Surg Sports Traumatol Arthrosc 14: 823-833.

2. Daikuya S, Suzuki T, Yabe K (2008) Neuromuscular function after reconstruction of anterior cruciate ligament--a case study using evoked electromyography. Electromyogr Clin Neurophysiol 48: 131-137.

3. Daikuya S, Ono A, Suzuki T, Yabe K (2009) Silent period and H reflex from soleus muscle as an index in a neuro-muscular function after reconstruction of anterior cruciate ligament. Electromyogr Clin Neurophysiol 49: 177-186.

4. Hoffmann P (1919) Demonstration of an inhibition of spinal cord reflexes Simmen wiles. Z Biol 70: 515-524.

5. Hugon M (1973) Methodology of the Hoffmann Reflex in Man. New Development in Electromyography and Clinical Neurophysiology 3: pp277-293.

6. Misiaszek JE (2003) The H-reflex as a tool in neurophysiology: its limitations and uses in understanding nervous system function. Muscle Nerve 28: 144160 .

7. Hall RC, Nyland J, Nitz AJ, Pinerola J, Johnson DL (1999) Relationship between ankle invertor $\mathrm{H}$-reflexes and acute swelling induced by inversion ankle sprain J Orthop Sports Phys Ther 29: 339-344.

8. Aagaard P, Simonsen EB, Andersen JL, Magnusson P, Dyhre-Poulsen $P$ (2002) Neural adaptation to resistance training: changes in evoked V-wave and H-reflex responses. J Appl Physiol 92: 2309-2318.

9. Sefton JM, Hicks-Little CA, Koceja DM, Cordova ML (2007) Effect of inversion and ankle bracing on peroneus longus Hoffmann reflex. Scand J Med Sci Sports 17: 539-546.

10. Burke D, Hallet M, Fuhr P (1999) Pierrot-Deseilligny E: $H$ reflexes from the tibial and median nerves. Recommendations for the practice of clinical neurophysiology; Guidelines of the international federation of clinical neurophysiology. Clin Neurophysiol, 52(supple): 259-262.

11. Roby-Brami A, Bussel B (1987) Long-latency spinal reflex in man after flexor reflex afferent stimulation. Brain $110: 707-725$.

12. Upton AR, McComas AJ, Sica RE (1971) Potentiation of "late" responses evoked in muscles during effort. J Neurol Neurosurg Psychiatry 34: 699-711.

13. Palmieri RM, Ingersoll CD, Hoffman MA, Cordova ML, Porter DA, et al. (2004) Arthrogenic muscle response to a simulated ankle joint effusion. $\mathrm{Br} \mathrm{J}$ Sports Med 38: 26-30

14. McVey ED, Palmieri RM, Docherty CL, Zinder SM, Ingersoll CD (2005) Arthrogenic muscle inhibition in the leg muscles of subjects exhibiting functional ankle instability. Foot Ankle Int 26: 1055-1061. 